\title{
Evidence of Fano-like resonances in mono-mode magnetic circuits
}

\author{
H. Al-Wahsh, ${ }^{1,2}$ E. H. El Boudouti, ${ }^{1,3}$ B. Djafari-Rouhani, ${ }^{1}$ A. Akjouj, ${ }^{1, *}$ T. Mrabti, ${ }^{3}$ and L. Dobrzynski ${ }^{1}$ \\ ${ }^{1}$ Institut d'Electronique, de Microélectronique et de Nanotechnologie (IEMN), UMR CNRS 8520, UFR de Physique, \\ Université des Sciences et Technologies de Lille, 59655 Villeneuve d'Ascq Cédex, France \\ ${ }^{2}$ Faculty of Engineering, Benha University, 11241 Cairo, Egypt \\ ${ }^{3}$ Laboratoire de Dynamique et d'Optique des Matériaux, Département de Physique, Faculté des Sciences, \\ Université Mohamed I, 60000 Oujda, Morocco \\ (Received 8 April 2008; revised manuscript received 9 June 2008; published 4 August 2008)
}

\begin{abstract}
In the frame of long-wavelength Heisenberg model, a simple magnonic device is designed to obtain possibly transmission stop bands (where the propagation of spin waves is forbidden). This simple device is composed of an infinite one-dimensional mono-mode waveguide (the backbone) along which $N\left(N^{\prime}\right)$ side resonators are grafted at two sites. Contrary to all known systems of this kind, a spectral transmission gap of nonzero width occurs here even with this simple structure. This is obtained by combining appropriately the zeros of transmission of the side resonators. Sharp resonant states inside the gaps can be achieved without introducing any defects in the structure. This results from an internal resonance of the structure when such a resonance is situated in the vicinity of a zero of transmission or placed between two zeros of transmission, the so-called Fano resonances. A general analytical expression for the transmission coefficient is given for various systems of this kind within the framework of the Green's function method. The amplitude, the phase, and the phase time of the transmission are discussed as a function of frequency and it is shown that the width of the stop bands is very sensitive to the number of the side resonators. These results should have important consequences for designing integrated devices such as narrow-frequency optical or microwave filters and high-speed switches.
\end{abstract}

DOI: 10.1103/PhysRevB.78.075401

PACS number(s): 75.30.Ds, 75.70.Cn, 75.90.+w

\section{INTRODUCTION}

Spin systems which have a regular distribution of scattering centers have been seen to possess a distinct and interesting array of magnonic properties, perhaps most strikingly frequency band gaps within which magnons cannot propagate through the structure - a so-called magnonic band gap. ${ }^{1-9}$ The interest in these band gaps is related to the potential applications of magnon-transport devices and is supported by the advanced progress in nanofabrication technology. ${ }^{10}$ Two-dimensional (2D) and three-dimensional (3D) composite systems constituted by periodic inclusions of at least two magnetic materials in a host matrix can exhibit an absolute magnonic band gap where the propagation of spin waves is inhibited in any direction of the space..$^{3,4,6,11-14}$ These magnonic band-gap materials can have many practical applications such as spin injection into devices. ${ }^{15}$ Studies of lower-dimensional systems such as one-dimensional (1D) periodic layered media ${ }^{2,16-20}$ and periodic waveguide systems with different geometries ${ }^{21-27}$ are conducted as analogs of 2D and 3D systems and for applications in their own right. These structures are attractive since their production is more feasible and they require only simple analytical and numerical calculations. On experimental grounds, arrays of very long ferromagnetic nanowires made of Ni Permalloy and Co with diameters in the range of 30-500 $\mathrm{nm}$ have been created. ${ }^{28}$ These are very uniform in cross section with lengths in the range of $20 \mu \mathrm{m}$. The realization of such nanowires can reasonably be considered, to an excellent approximation, as mono-mode waveguides.

In order to provide comprehensive information about magnetotransport in magnonic band-gap crystals (which is the analogous to photonic crystals and based on magnetic materials), in this paper, we propose a different structure and focus our study on it. This structure consists of $N\left(N^{\prime}\right)$ side resonators grafted at two sites on an infinite 1D mono-mode waveguide. The transmission gaps and Fano-like resonances have been established through an analysis of the transmission function (amplitude and phase) obtained within the framework of the Green's function method. In a previous publication, ${ }^{21-23}$ we reported that the magnonic transmission spectrum of 1D comb structures exhibits large gaps. These structures, called star wave guides, are composed of $M^{\prime}$ dangling side branches (DSBs) periodically grafted at each of the $M$ equidistant sites on an infinite 1D mono-mode waveguide (the backbone). The gaps originate from the periodicity of the system determined by the distance between two neighboring sites and from the eigenfrequencies of the DSB which play the role of resonators. The gap widths also depend on the effect of the pinning field at the ends of the side branches. ${ }^{23}$ It is interesting to underline the results of huge gaps and discrete transmission spectrum due only to the dangling side branches grafted at a single site on the backbone. Unlike other 1D (e.g., Bragg lattices), 2D, or 3D magnonic crystals in which the contrast between the constituents is a critical parameter for the stop band existence, this star waveguide exhibits relatively large forbidden bands even if the backbone and the resonators are made of the same material. On the other hand, in Refs. 24-27 we have presented a networks called a serial loop structure (SLS). These SLS was made of symmetric (asymmetric) loops pasted together with segments of finite length; the loops play the role of resonators. Such structure exhibits new features, in comparison with the star waveguide. We emphasize the interesting result of transmission zeros in the case of asymmetric loop 
structure. $^{26}$ These transmission zeros may lead to a phase drop of $\pi$ and therefore negative phase time. ${ }^{29}$

Recently magnonic band-gap crystals, in which propagation of spin waves (magnons) is forbidden, have attracted much attention. This is related to a number of advantages that magnonic crystals have in comparison with photonic crystals. The wavelength of a spin wave and, hence, the properties of such crystals depend on the external magnetic field and can be controlled by this field. The wavelength of propagating spin waves for a wide class of ferromagnetic materials in the microwave range is on the order of tens or even hundreds of micrometers. The phase and group velocities of spin waves are also functions of the structure size and the applied external field and may vary over a wide range. ${ }^{1}$ In addition to the band gaps, a great interest has been paid to the so-called Fano resonances that may be introduced in such gaps. Some analytical models, in phononic band-gap crystals, have been proposed to explain the origin and the behavior of such resonances. ${ }^{30-33}$ These resonances were first theoretically described by Fano $^{34}$ when he studied the inelastic auto ionizing resonances in atoms. The asymmetry (Fano profile) was explained as the result of the interference between the discrete resonances with the smooth continuum background in which the former is embedded. The symmetric and asymmetric line shapes have been also reported in the electronic transport in mesoscopic systems using the Aharonov-Bohm systems. ${ }^{35-38}$ Mainly, the subject of these studies was to use these interferometric systems to show the conditions for the existence and the collapse of Fano resonances as a function of the applied current-voltage and magnetic flux. These studies are also related to the investigation of the electronic states of quantum dots ${ }^{35,36}$ as well as to the understanding ${ }^{29,39}$ of the transmission phase jumps by $\pi$ between two adjacent resonances in relation with the experiments of Yacoby et al. ${ }^{40}$ The analogy between scattering properties of electrons, phonons, and magnons suggests that this type of feature can also appear in magnonic systems. ${ }^{41}$

The motivation behind the work presented in this paper is to introduce a design of a simple magnonic filter consisting of $N\left(N^{\prime}\right)$ dangling side branches, which play the role of resonators, grafted at two sites on an infinite 1D mono-mode waveguide [see the inset of Figs. 1(a), 1(c), and 1(e)]. We show analytically and numerically that this simple structure can exhibit transmission gaps (their width depend on the number of dangling resonators) and Fano-like resonances. In particular, we show that the transmission amplitude through such a system can be written following the Fano-like shape around these resonances. In addition, we give an explicit expression of the Fano parameter ${ }^{34}$ as well as the position and the width of the Fano resonances ${ }^{34}$ as a function of the geometrical parameters of the system.

This paper is organized as follows. In Sec. II, we give a brief review of the theoretical model used in this work as well as the analytical results of the structure depicted above. These results are necessary for an analytical understanding of the phenomenon obtained for the structure proposed in this work. Section III is devoted to the transmission gaps and Fano resonances. The conclusions are presented in Sec. IV.

\section{THEORETICAL DISCUSSION}

We consider an infinite (one dimensional) ferromagnetic medium $i$. The Fourier-transformed Green's function between two points $x$ and $x^{\prime}$ of this infinite waveguide can be expressed as ${ }^{21,42}$

$$
G_{i}\left(x, x^{\prime}\right)=-\frac{e^{-\alpha_{i}\left|x-x^{\prime}\right|}}{2 F_{i}},
$$

where $\alpha_{i}=j \sqrt{\left(\omega-\gamma_{i} H_{0}\right) / D_{i}^{\prime}}=j \alpha_{i}^{\prime}, \quad j=\sqrt{-1}, \quad F_{i}=D_{i}^{\prime} \alpha_{i} / \gamma_{i} M_{0 i}$, and $D_{i}^{\prime}=\left(2 J a^{2} M_{0 i}\right) /\left(\gamma_{i} \hbar^{2}\right) . \omega, M_{0 i}, H_{0}, J$, and $\gamma_{i}$ stand, respectively, for the angular frequency of the spin wave, the spontaneous magnetization, the static external field, the exchange interaction between neighboring magnetic sites in the simple-cubic lattice of lattice parameter $a$ constituting the ferromagnetic medium, and the gyromagnetic ratio. Before addressing the problem of the simple structure presented in this work [see the inset of Figs. 1(a), 1(c), and 1(e)], it is helpful to know the surface elements of its elementary constituents, namely, the Green's function of a finite segment of length $d_{i}, i=1,2,3$ and of a semi-infinite medium (lead). The finite segment of length $d_{2}$ is bounded by two free surfaces located at $x=0$ and $x=d_{2}$. These surface elements can be written in the form of a $(2 \times 2)$ matrix $g_{2}(M M)$ within the interface space $M=\left\{0,+d_{2}\right\}$. The inverse of this matrix takes the following form: ${ }^{43}$

$$
g_{2}^{-1}(M M)=\left(\begin{array}{cc}
\frac{-F_{2} C_{2}}{S_{2}^{\prime}} & \frac{F_{2}}{S_{2}^{\prime}} \\
\frac{F_{2}}{S_{2}^{\prime}} & \frac{-F_{2} C_{2}}{S_{2}^{\prime}}
\end{array}\right),
$$

where $C_{2}=\cos \left(\alpha_{2}^{\prime} d_{2}\right)$ and $S_{2}^{\prime}=j \sin \left(\alpha_{2}^{\prime} d_{2}\right)=j S_{2}$. The inverse of the surface Green's functions of the dangling resonators grafted at the sites $\{0\}$ and $\left\{d_{2}\right\}$ is given by $g_{1}^{-1}(0,0)$ $=-N F_{1} C_{1} / S_{1}^{\prime}$ and $g_{3}^{-1}\left(d_{2}, d_{2}\right)=-N^{\prime} F_{3} C_{3} / S_{3}^{\prime}$, where $C_{i}$ $=\cos \left(\alpha_{i}^{\prime} d_{i}\right), S_{i}^{\prime}=j \sin \left(\alpha_{i}^{\prime} d_{i}\right)=j S_{i}, i=1,3 . N$ and $N^{\prime}$ are the number of side branches on both sides of the finite segment of length $d_{2}$. The inverse of the surface Green's functions of the two semi-infinite ferromagnetic leads surrounding the whole structure is given by $g_{s}^{-1}(0,0)=g_{s}^{-1}\left(d_{2}, d_{2}\right)=-F_{s}$. In what follows, we assume that the semi-infinite leads and mediums (segments) 1, 2, and 3 are constituted of the same material [i.e., $F_{1}=F_{2}=F_{3}=F_{s}=F=D^{\prime} \alpha / \gamma M_{0}$, $D^{\prime}=\left(2 J a^{2} M_{0}\right) /\left(\gamma \hbar^{2}\right)$, and $\left.\alpha=j \sqrt{\left(\omega-\gamma H_{0}\right) / D^{\prime}}=j \alpha^{\prime}\right]$. We report on results of calculated transmission coefficients and phase or phase time as a function of frequency. Using the Green's function method, ${ }^{43}$ the expression giving the inverse of the Green's function of the whole system [depicted at the inset of Fig. 1(e) for $N=N^{\prime}=2$ ] can be obtained from a linear superposition of the above inverse Green's functions of the constituent, namely,

$$
g^{-1}(M M)=F\left(\begin{array}{cc}
\frac{-C_{2}}{S_{2}^{\prime}}-\frac{N S_{1}^{\prime}}{C_{1}}-1 & \frac{1}{S_{2}^{\prime}} \\
\frac{1}{S_{2}^{\prime}} & \frac{-C_{2}}{S_{2}^{\prime}}-\frac{N^{\prime} S_{3}^{\prime}}{C_{3}}-1
\end{array}\right) .
$$



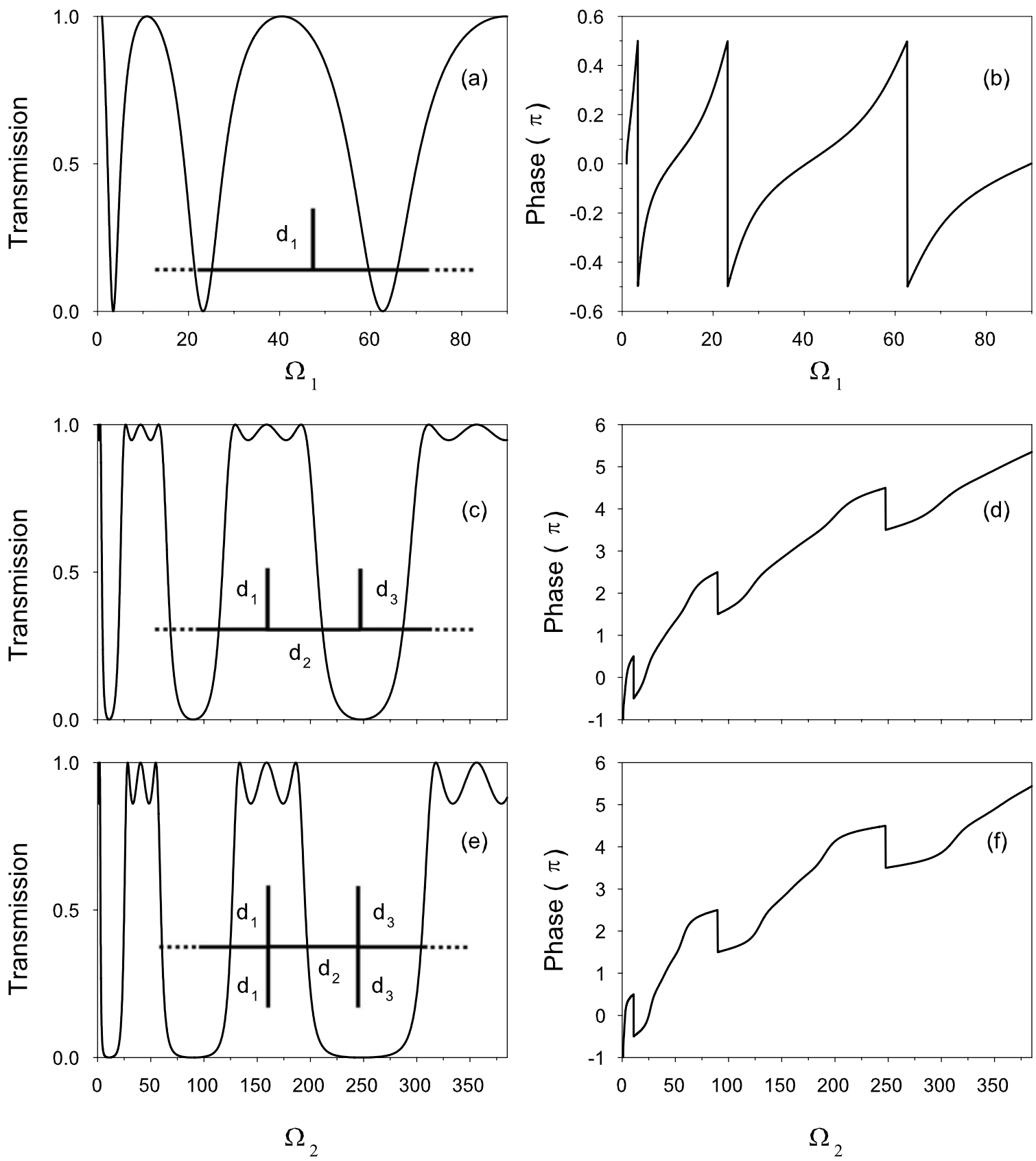

FIG. 1. (a) Transmission coefficient vs the reduced frequency $\Omega_{1}$ for the structure depicted in the inset. For convenience $\widetilde{H}^{\prime}$ is considered to be 1. (c) Transmission coefficient vs the reduced frequency $\Omega_{2}$ for the structure depicted in the inset with $d_{1}=d_{3}=0.5 d_{2}$ and $N=N^{\prime}=1$. (e) The same as in (c) but for the structure depicted in the inset of (e) $\left(N=N^{\prime}=2\right)$. (b), (d), and (f) are, respectively, the same as (a), (c), and (e) but for the variation of the phase.

The transmission function is given by ${ }^{43} t=-2 F g\left(0, d_{2}\right)$ or equivalently

$$
t=\frac{2 C_{1} C_{3}}{\beta_{1}+j \beta_{2}}
$$

where

$$
\beta_{1}=2 C_{1} C_{2} C_{3}-S_{2}\left(N S_{1} C_{3}+N^{\prime} C_{1} S_{3}\right),
$$

and

$$
\beta_{2}=2 C_{1} S_{2} C_{3}+N^{\prime} C_{1} C_{2} S_{3}+N S_{1}\left(C_{2} C_{3}-N^{\prime} S_{2} S_{3}\right) .
$$

From the expression of $t$ [Eq. (4)], one can deduce the transmission amplitude

$$
T=\frac{4 C_{1}^{2} C_{3}^{2}}{\beta_{1}^{2}+\beta_{2}^{2}},
$$

as well as the phase 


$$
\varphi=\arctan \left(\beta_{2} / \beta_{1}\right)+\pi \Theta\left[C_{1} C_{3}\right],
$$

where $\Theta$ means the Heaviside function. From Eqs. (4) and (7) one can notice that the transmission zeros are induced by the side branches (i.e., $C_{1}=0$ or $C_{3}=0$ ). When the expression $C_{1} C_{3}$ changes sign at some frequencies denoted by $\omega_{n}$, then the phase [Eq. (8)] exhibits a jump of $\pi$.

Another interesting quantity is the first derivative of $\varphi$ with respect to the pulsation $\omega$ which is related to the delay time taken by the magnons to traverse the structure. This quantity, called phase time, is defined by ${ }^{44,45}$

$$
\tau_{\varphi}=\frac{d \varphi}{d \omega}
$$

and can be written as

$\tau_{\varphi}=\frac{d}{d \omega} \arctan \left(\beta_{2} / \beta_{1}\right)+\pi \sum_{n} \operatorname{sgn}\left[\frac{d}{d \omega}\left(C_{1} C_{3}\right)_{\omega=\omega_{n}}\right] \delta\left(\omega-\omega_{n}\right)$,

where sgn means the sign function. Furthermore, the density of states (DOS) of the present composite system from which we have subtracted the DOS of the semi-infinite leads is given by ${ }^{45}$

$$
\Delta n(\omega)=\frac{1}{\pi} \frac{d}{d \omega} \arctan \left(\beta_{2} / \beta_{1}\right) .
$$

Because of the second term on the right-hand side of Eq. (10), one can deduce that $\tau_{\varphi} \neq \pi \Delta n(\omega)$ as $\tau_{\varphi}$ [Eq. (10)] may exhibit $\delta$ functions at the transmission zeros that do not exist in the variation of the DOS [Eq. (11)]. However, if the system does not exhibit transmission zeros, then $\Theta\left[C_{1} C_{3}\right]=0$ and $\tau_{\varphi}=\pi \Delta n(\omega)$. It should be pointed out that the validity of our results is subject to the requirement that the cross section of the waveguide being negligible compared to their length and to the propagation wavelength. The assumption of mono-mode propagation is then satisfied.

\section{TRANSMISSION GAPS AND FANO RESONANCES}

Before addressing the problem of the whole structure described above, let us first recall briefly the results of a particular case necessary for the understanding of the spin-wave propagation in the structures shown at the inset of Figs. 1(c) and 1(e), namely, if $d_{2}=0, N=1$ and $N^{\prime}=0$, we obtain the transmission function of a simple structure consisting of one resonator grafted on an infinite guide [see the inset of Fig. 1(a)]: $t=C_{1} /\left(C_{1}+j S_{1} / 2\right)$. This expression enables us to deduce the transmission coefficient $T=|t|^{2}=4 C_{1}^{2} /\left(4 C_{1}^{2}+S_{1}^{2}\right)$ and the phase $\varphi=\pi \Theta\left(C_{1}\right)-\arctan \left(S_{1} / 2 C_{1}\right)$. Let us mention that the transmission amplitude of a quantum waveguide with one resonator has been previously discussed in numerous publications. ${ }^{46}$ We can see that the transmission coefficient equals zero when $C_{1}=0$, i.e., $\alpha^{\prime} d_{1}=\left(l^{\prime}+0.5\right) \pi$, where $l^{\prime}$ is a positive integer. The corresponding frequencies will be $\omega_{g}$ $=\gamma H_{0}+D^{\prime}\left[\left(l^{\prime}+0.5\right) \pi / d_{1}\right]^{2}$ or symbolically $\Omega_{g}=\widetilde{H}^{\prime}+\left[\left(l^{\prime}\right.\right.$ $+0.5) \pi]^{2}$, where $\Omega_{g}=\omega_{g} d_{1}^{2} / D^{\prime}$ is a reduced frequency and $\tilde{H}^{\prime}=\gamma H_{0} d_{1}^{2} / D^{\prime}$. From these results one can notice that for this composite system there exist an infinite set of forbidden frequencies $\Omega_{g}$ corresponding to the eigenmodes of the grafted finite branch. This grafted branch behaves as a resonator and this simple composite system filters out the modes $\Omega_{g}$. This phenomenon is related to the resonances associated with the finite additional path offered to the spin-wave propagation. The variation of $T$ versus the reduced frequency $\Omega_{1}=\omega d_{1}^{2} / D^{\prime}$ is reported in Fig. 1(a). $T$ is equal to zero when $\alpha^{\prime} d_{1}$ is an odd multiple of $\pi / 2$ and reaches its maximum value of 1 when $\alpha^{\prime} d_{1}$ is a multiple of $\pi$. The variation in the phase versus the reduced frequency [Fig. 1(b)] shows an abrupt change in $\pi$ at the transmission zeros and therefore the corresponding phase time is different from the DOS as mentioned above [Eqs. (10) and (11)].

For the structures shown at the insets of Figs. 1(c) and 1(e), Eq. (4) clearly shows that the transmission zeros are due only to the dangling resonators (i.e., when $C_{1}=0$ or $C_{3}$ $=0)$. Figure 1(c) gives the transmission coefficient in presence of two identical dangling resonators (i.e., $N=N^{\prime}=1$ and $d_{1}=d_{3}=0.5 d_{2}$ ). One can notice that the transmission coefficient presents well-defined dips induced by the grafted branches. This dip transform into large transmission gap when the number of branches increase as it is illustrated in Fig. 1(e) for $N=N^{\prime}=2$. It is worth mentioning that because of the existence of two resonators, one can expect two phase drops of $\pi$ (i.e., $2 \pi$ ) at the transmission zeros given by $C_{1}=C_{3}=0$ [i.e., $\Omega_{2}=\omega d_{2}^{2} / D^{\prime}=\widetilde{H}+\left[\left(l^{\prime}+0.5\right) \pi\right]^{2}, \widetilde{H}$ $\left.=\gamma H_{0} d_{2}^{2} / D^{\prime}, l^{\prime}=0,1,2, \ldots\right]$. However, one can see in Figs. 1(d) and 1(f) that the phase presents only a phase drop of $\pi$. This is due to existence of a resonant state with zero width at these values of $\Omega_{2}$ which induce a phase jump of $\pi$; these resonances collapse when $d_{1}=d_{3}$ is taken exactly equal to $0.5 d_{2}$. To enlarge these resonances, we have to take $d_{1}$ and $d_{3}$ slightly different from $0.5 d_{2}$. Indeed, at $\Omega_{2}=\widetilde{H}+(l \pi)^{2}, l$ $=1,2, \ldots$ and for $N=N^{\prime}$, the expression of the transmission function [Eq. (4)] becomes

$$
t= \pm \frac{2 C_{1} C_{3}}{2 C_{1} C_{3}+j N \sin \left[\alpha^{\prime}\left(d_{1}+d_{3}\right)\right]} .
$$

In particular, if $\alpha^{\prime}\left(d_{1}+d_{3}\right)=m \pi, \alpha^{\prime} d_{1} \neq\left(m_{1}+0.5\right) \pi$, and $\alpha^{\prime} d_{3} \neq\left(m_{2}+0.5\right) \pi\left(m, m_{1}\right.$, and $m_{2}$ are integers), one obtains a resonance that reaches unity (i.e., $T=1$ ). An example corresponding to this situation is given in Fig. 2(a) where $N$ $=N^{\prime}=1, d_{1}=0.46 d_{2}$, and $d_{3}=0.54 d_{2}\left(\right.$ with $\left.d_{1}+d_{3}=d_{2}\right)$. One can notice that the resonance at $\Omega_{2}=\widetilde{H}+\pi^{2}$ is squeezed between two zeros [indicated by solid circles on the abscissa of Fig. 2(a)] induced by the dangling resonators as it is also illustrated in the plot describing the variation of the phase [Fig. 2(c)]. The width of this resonance increases as far as $d_{1}$ and $d_{3}$ deviate from $0.5 d_{2}$ (see below). In the particular case where $\alpha^{\prime} d_{1}=\left(m_{1}+0.5\right) \pi$ and $\alpha^{\prime} d_{3}=\left(m_{2}+0.5\right) \pi$, the numerator and denominator of $t$ [Eq. (12)] vanishes altogether. In this case, the resonance as well as the two zeros induced by the resonators fall at the same position, then the resonance collapses, the transmission coefficient vanishes and the phase drops by $\pi$ as it was shown in Figs. 1(d) and 1(f).

The resonance in Fig. 2(a) shows the same characteristics as a Fano resonance but with two zeros of transmission 

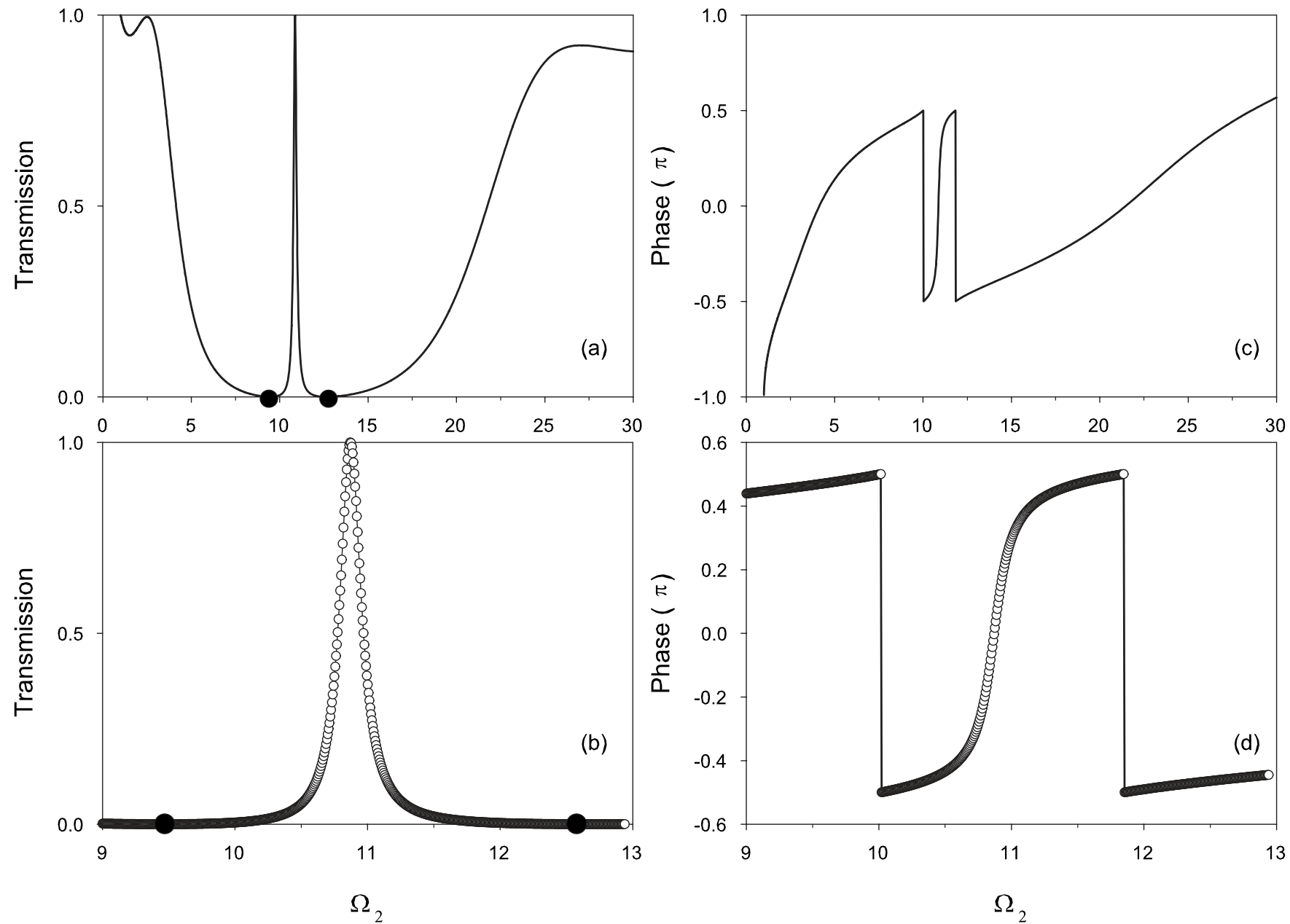

FIG. 2. (a) The same as in Fig. 1(c) but the lengths of the resonators are taken such that $d_{1}=0.46 d_{2}$ and $d_{3}=0.54 d_{2}$ and $N=N^{\prime}=1$. Solid circles on the abscissa indicate the positions of the transmission zeros induced by the dangling resonators on both sides of the resonance. (c) The same as in (a) but for the variation of the phase. (b) and (d) give the approximate results (open circles) around the resonance.

around the resonance instead of one as it is usually the case. ${ }^{34,35}$ Indeed, one can obtain an approximate analytical expression for the transmission function [Eq. (4)] in the vicinity of the resonance. A Taylor expansion around $\alpha^{\prime} d_{2}$ $=\pi$ (i.e., $\alpha^{\prime} d_{2}=\pi+\varepsilon$ with $\varepsilon / \pi \ll 1$ and $N=N^{\prime}$ ) enables us to obtain

$$
t=\frac{-\zeta \zeta^{\prime}}{2 N \varepsilon^{2}+\zeta \zeta^{\prime}-j \varepsilon\left[2\left(N+N^{2}\right)+\zeta \zeta^{\prime}\left(N^{2}-2\right) / 2\right]} .
$$

where $\zeta=2 \Delta+\varepsilon(1+2 \Delta / \pi), \zeta^{\prime}=-2 \Delta+\varepsilon(1-2 \Delta / \pi)$, and $\Delta$ is the detuning of $d_{1}$ and $d_{3}$ from $0.5 d_{2}$ [i.e., $\Delta=\pi(0.5$ $\left.\left.-d_{1} / d_{2}\right)=\pi\left(-0.5+d_{3} / d_{2}\right)\right]$. Using Eq. (13), one can show that the transmission coefficient $T$ can be written (following the Fano line shape ${ }^{34,35}$ ) in the form

$$
T=A \frac{\left(\varepsilon+q_{1} \Gamma\right)^{2}\left(\varepsilon-q_{2} \Gamma\right)^{2}}{\varepsilon^{2}+\Gamma^{2}},
$$

where $A=\left(1-4 \Delta^{2} / \pi^{2}\right)^{2} / 4\left[N(N+1)+\Delta^{2}\left(2-N^{2}\right)\right]^{2}$.

$$
\Gamma=2 \Delta^{2} /\left[N(N+1)+\Delta^{2}\left(2-N^{2}\right)\right]
$$

characterizes the width of the resonance falling at $\varepsilon=0$.

$$
q_{1}=\left[N(N+1)+\Delta^{2}\left(2-N^{2}\right)\right] / \Delta(1+2 \Delta / \pi)
$$

and

$$
q_{2}=\left[N(N+1)+\Delta^{2}\left(2-N^{2}\right)\right] / \Delta(1-2 \Delta / \pi)
$$

are the coupling parameters; they give qualitatively the interference between the bound states and the propagating continuum states. ${ }^{34-36}$

One can notice that when increasing $\Delta, \Gamma$ increases and $q_{1}\left(q_{2}\right)$ decreases. The results of the approximate expression [Eq. (14)] are shown in Fig. 2(b) by open circles. These results are in accordance with the exact ones (solid lines) and clearly show that the resonance is of Fano type with $q_{1}$ $\simeq 14.85$ and $q_{2} \simeq 17.43$ and width $2 \Gamma \simeq 0.03$. The commonly studied Fano resonances are asymmetric because of the presence of only one transmission zero near the resonance (see below). In addition, in the electronic counterparts studies, a perturbation is often introduced to the system in order to create the resonance state. ${ }^{34-37}$ However, the above calculation shows that, without introducing any perturbation in the structure, one can find a well-defined symmetric Fano resonance with width $2 \Gamma$ and coupling parameters $q_{1}$ and $q_{2}$ that 

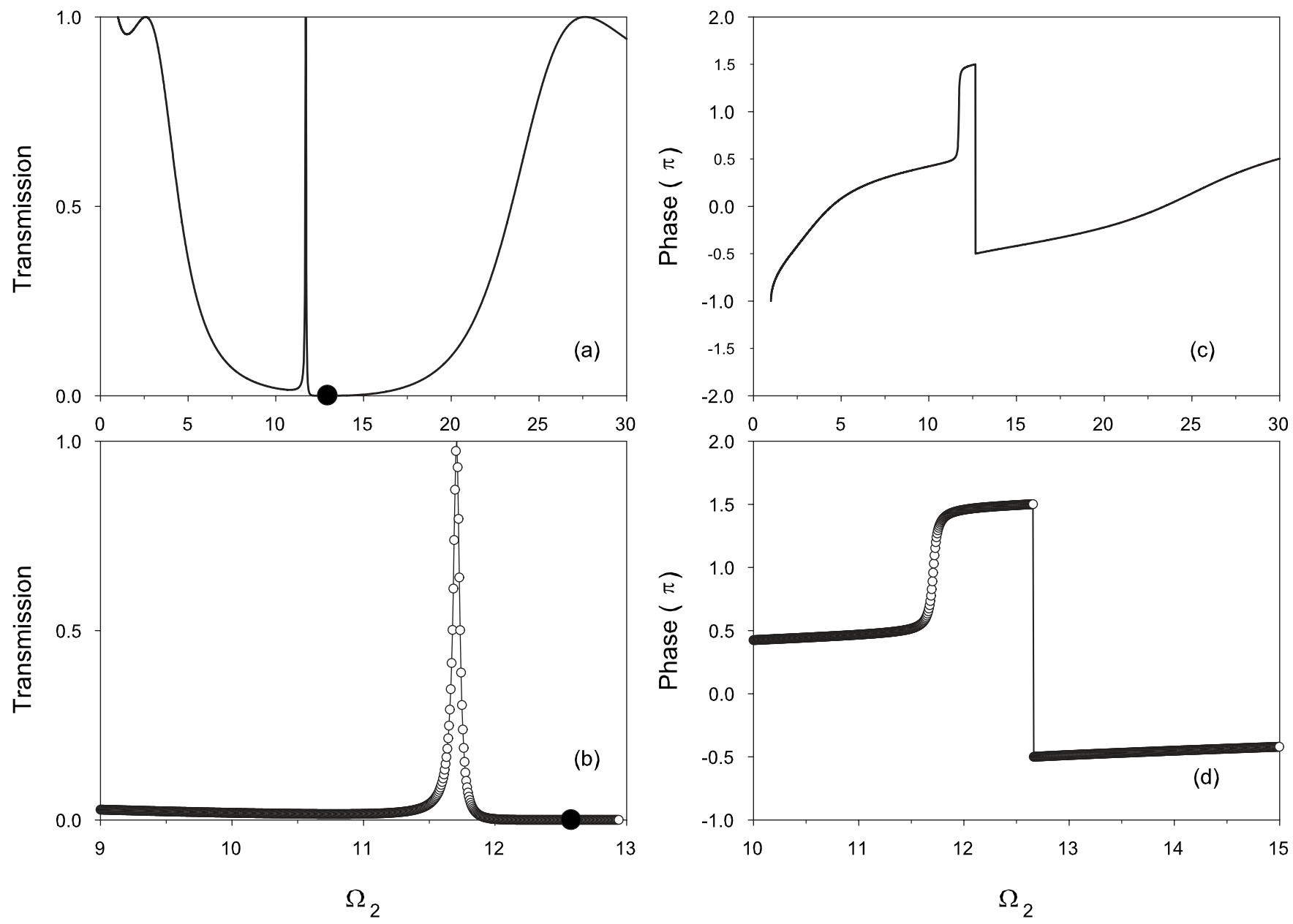

FIG. 3. (a) The same as in Fig. 2(a) but the resonators are taken to be of identical lengths $d_{1}=d_{3}=0.46 d_{2}$, (c) The same as in (a) but for the variation of the phase. (b) and (d) give the approximate results (open circles) around the resonance.

can be adjusted by tailoring the lengths of the resonators (i.e., $\Delta$ ). Equation (13) enables us also to deduce an approximate expression for the phase as

$$
\begin{aligned}
\varphi= & -\arctan \left\{\frac{\varepsilon\left[2\left(N+N^{2}\right)+\zeta \zeta^{\prime}\left(N^{2}-2\right) / 2\right]}{2 N \varepsilon^{2}+\zeta \zeta^{\prime}}\right\} \\
& +\pi \Theta(\zeta)+\pi \Theta\left(\zeta^{\prime}\right) .
\end{aligned}
$$

This function is plotted by open circles in Fig. 2(d) and clearly shows two abrupt phase changes in $\pi$ at $\zeta=0$ and $\zeta^{\prime}=0$ (i.e., $\varepsilon_{1}=-q_{1} \Gamma$ and $\varepsilon_{2}=q_{2} \Gamma$ ) in accordance with the exact results (solid line).

One can also create an asymmetric Fano resonance by adjusting the transmission zeros on only one side of the resonance; this can be obtained by considering a structure where the resonators are supposed to be identical with lengths slightly different from $0.5 d_{2}$. This is shown in Fig. 3(a) for $d_{1}=d_{3}=0.46 d_{2}$ and $N=N^{\prime}=1$. Indeed, an analytical Taylor expansion around $\alpha^{\prime} d_{2}=\pi$ enables us to write the transmission function [Eq. (4)] as

$$
t=\frac{-2 \xi^{2}}{(\xi-j N)(N \varepsilon+2 \xi+j \varepsilon \xi)},
$$

where $\xi=\Delta+0.5 \varepsilon(1+2 \Delta / \pi)$ and $\Delta$ is the detuning of the lengths of the two resonators from $0.5 d_{2}$ [i.e., $\Delta=\pi\left(d_{1} / d_{2}\right.$
$-0.5)]$. From the expression of $t$ [Eq. (19)], one can deduce the following Fano line-shape transmission coefficient:

$$
T=\frac{B}{N^{2}+\xi^{2}} \frac{\left(\varepsilon-\varepsilon_{R}+q \Gamma\right)^{4}}{\left(\varepsilon-\varepsilon_{R}\right)^{2}+\Gamma^{2}} \simeq \frac{B}{N^{2}} \frac{\left(\varepsilon-\varepsilon_{R}+q \Gamma\right)^{4}}{\left(\varepsilon-\varepsilon_{R}\right)^{2}+\Gamma^{2}},
$$

where $B=(1+2 \Delta / \pi)^{4} / 4(N+1+2 \Delta / \pi)^{2}$.

$$
q=(N+1+2 \Delta / \pi)^{2} / \Delta(1+2 \Delta / \pi)
$$

is the Fano parameter.

$$
\Gamma=2 \Delta^{2} / N^{2}\left[1+\frac{1}{N}(1+2 \Delta / \pi)\right]^{3}
$$

and

$$
\varepsilon_{R}=-2 \Delta /(N+1+2 \Delta / \pi)
$$

characterize the width and the shift of the resonance, respectively.

One can notice that the resonance shifts slightly from $\alpha^{\prime} d_{2}=\pi$ and its width is small as compared to the preceding case; this is in accordance with the numerical results of Figs. 2(a) and 3(a). Also $q$ increases when $\Delta$ decreases and tends to infinity when $\Delta$ vanishes. In this case the resonance falls at $\varepsilon_{R}=0$ and, as expected, its width $2 \Gamma$ reduces to zero [see 

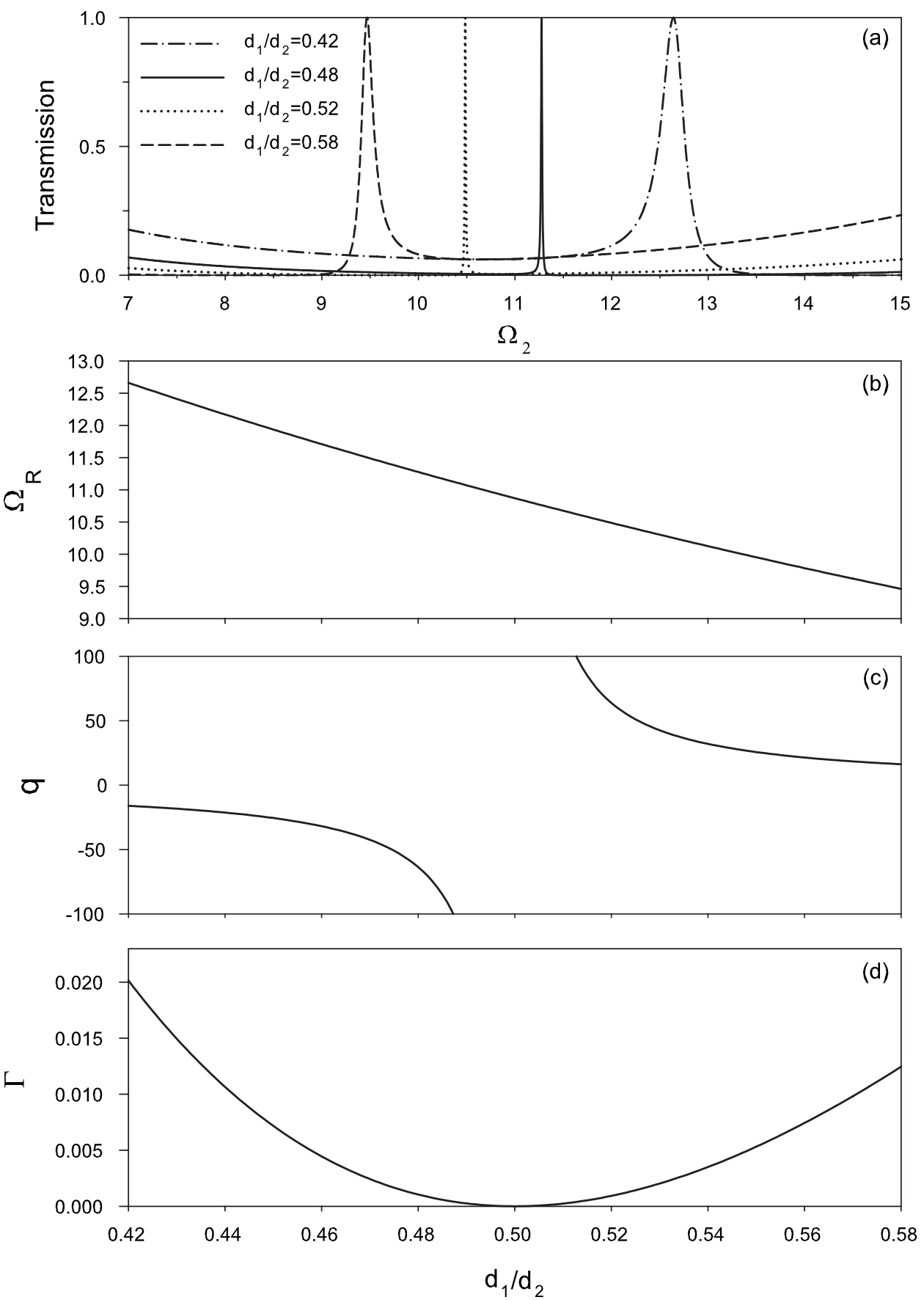

FIG. 4. (a) The same as in Fig. 3(a) but for different values of $d_{1} / d_{2}$. [(b)-(d)] Variations of the quantities $\left[\Omega_{R}=\tilde{H}+\left(\pi+\varepsilon_{R}\right)^{2}, \tilde{H}\right.$ $=1$ ] [Eq. (23)], $q$ [Eq. (21)], and $\Gamma$ [Eq. (22)] as a function of $d_{1} / d_{2}$ around $d_{1} / d_{2}=0.5$.
Fig. 1(c)]. The results of the approximate expression [Eq. (20)] are sketched (open circles) in Fig. 3(b) for $\Delta$ $=\pi\left(d_{1} / d_{2}-0.5\right)=-0.04 \pi$ (i.e., $d_{1} / d_{2}=0.46$ ) and $N=N^{\prime}=1$.

These results are in accordance with the exact ones (solid lines) and clearly show that the resonance is of Fano type with $|q| \simeq 32$ and width $2 \Gamma \simeq 0.0089$. Concerning the evolution of the phase of the spin waves in this structure, one can notice from Eq. (4) that the numerator of the transmission function $t$ vanishes when $C_{1}=C_{3}=0$ [or equivalently $\xi=0$ in the approximate result [Eq. (19)] at $\alpha^{\prime} d_{2}=(\pi / 2) d_{2} / d_{1}$ (i.e., $\left.\Omega_{2}=12.66\right)$ indicated by a filled circle on the abscissa of Fig. 3(a)]. The transmission zeros induced by the two identical resonators fall at the same frequency; therefore the phase [Figs. 3(c) and 3(d)] shows a phase drop of $2 \pi$ at these frequencies. Indeed, as the phase is defined $\bmod 2 \pi$, the $2 \pi$ phase change can be observed if we take into account the absorption in the system. ${ }^{47,48}$

In order to show the profile of the Fano resonances as a function of the parameter $\Delta$ (or equivalently $d_{1} / d_{2}$ ), Fig. 4(a) gives the characteristic features of the resonances as a function of the reduced frequency $\Omega_{2}$ for $d_{1} / d_{2}$ around 0.5 and for an asymmetric resonance. On can notice that the position of the resonance decreases as a function of $d_{1} / d_{2}$; its asymmetric Fano profile becomes symmetric and changes sign for $d_{1} / d_{2} \simeq 0.5$. In other words, the parameter $q$ responsible for the asymmetric Fano profile of the resonance diverges and changes sign around $d_{1} / d_{2}=0.5$. The width of the resonance decreases when $d_{1} / d_{2}$ tends to 0.5 and vanishes when $d_{1} / d_{2}$ 


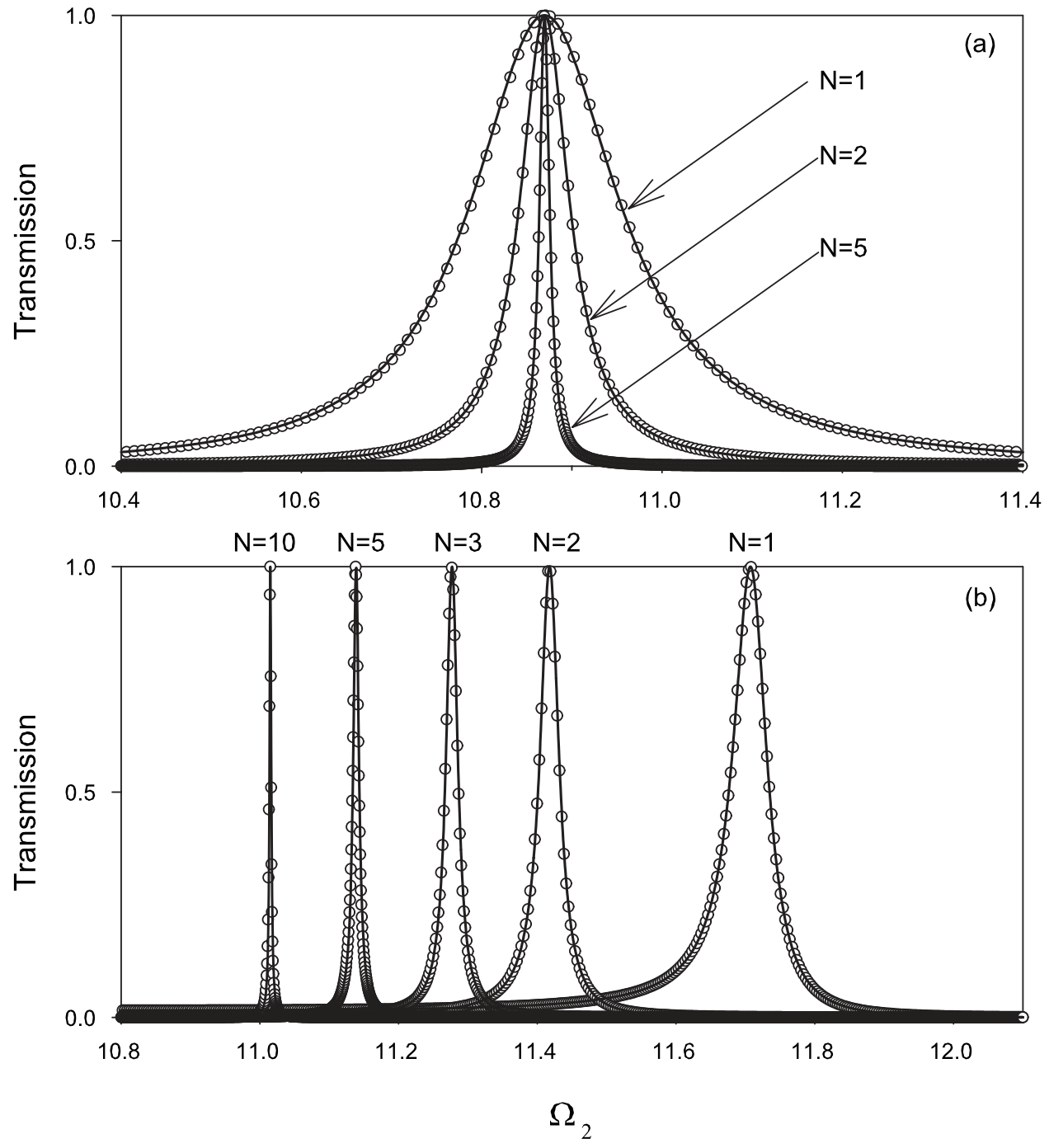

FIG. 5. (a) The Fano profile of the symmetric resonance depicted in Fig. 2(a) for different values of $N=N^{\prime}$. (b) The same as in (a) but for the asymmetric Fano resonance depicted in Fig. 3(a). is exactly equal 0.5 giving rise to the collapse of the resonance [see Fig. 4(a)]. These results are well illustrated by the plots of the approximate expressions of $\Omega_{R}=\tilde{H}+\left(\pi+\varepsilon_{R}\right)^{2}$ [Fig. 4(b)], $q$ [Fig. 4(c)], and $\Gamma$ [Fig. 4(d)] around $d_{1} / d_{2}$ $=0.5$.

Until now we have concentrated our analysis on the waveguide structure with only one dangling resonator (i.e., $N=N^{\prime}=1$ ). The advantage of such a structure lies in the facility to be designed experimentally. However, the analytical approximate expressions [Eqs. (14)-(17) and (20)-(23)] clearly show that the resonances remain of Fano type even for $N=N^{\prime}$ different from one. Indeed, Fig. 5 gives the dependence of the transmission rate of both symmetric [Fig. 5(a)] and asymmetric [Fig. 5(b)] Fano resonances for different values of $N=N^{\prime}$. Figure 5(a) displays the transmission amplitude $T$ [Eq. (7)] as a function of $\Omega_{2}$ for $N=N^{\prime}=1,2$, and 5; the other parameters are $d_{1}=0.46 d_{2}, d_{3}=0.54 d_{2}$, and $\tilde{H}=1$. The results of the approximate expression [Eq. (14)] are sketched by open circles. Even though the resonances fall at the same frequency $\Omega_{g} \simeq 10.88$ (i.e., $\alpha_{2}^{\prime} d_{2}=\pi$ ), their widths decrease as a function of $N$ and their $q$ parameter increases giving rise to a symmetric resonance of Breight-Wigner type. These results are in accordance with the approximate results of Eqs. (14)-(17).
In the same way, Fig. 5(b) depicts the effect of variation in the number $N$ of DSBs on the transmission rate $T$ for a structure constituted of two identical DSBs with $d_{1}=d_{3}$ $=0.46 d_{2}$. One can notice that, contrary to the results of Fig. 5 (a), the position of the resonance decreases as a function of $N$ and tends to $\Omega_{2} \simeq 10.88$ (i.e., $\alpha_{2}^{\prime} d_{2}=\pi$ ) when $N$ goes to infinity [see Eq. (23)]. However, the width $\Gamma$ of the resonance and its $q$ parameter exhibit similar behavior as in Fig. 5(a) when $N$ increases [see Eqs. (21) and (22)].

In Fig. 6(a), the transmission rate through the structures shown at the insets of Fig. 1(c) $\left(N=N^{\prime}=1\right.$, solid line) and Fig. 1(e) $\left(N=N^{\prime}=2\right.$, dashed line) is redrawn for the sake of comparison with Fig. 6(b) giving the variation of the DOS. The lengths of the resonators are chosen such that $d_{1}=d_{3}$ $=0.5 d_{2}$. A well-defined gap is obtained when the number of resonators is increased. Such a stop band could be useful in constructing a rejecting signal device. In Fig. 6(b), one can notice that the DOS is strongly reduced in the transmission gap regions, in particular, when the number of dangling resonators increases. An analysis of the phase time is given in Fig. 6(c). This quantity gives information on the time spent by the magnon inside the structure before its transmission. Because of the existence of the transmission zeros, the phase time gives rise to delta functions around the transmission 

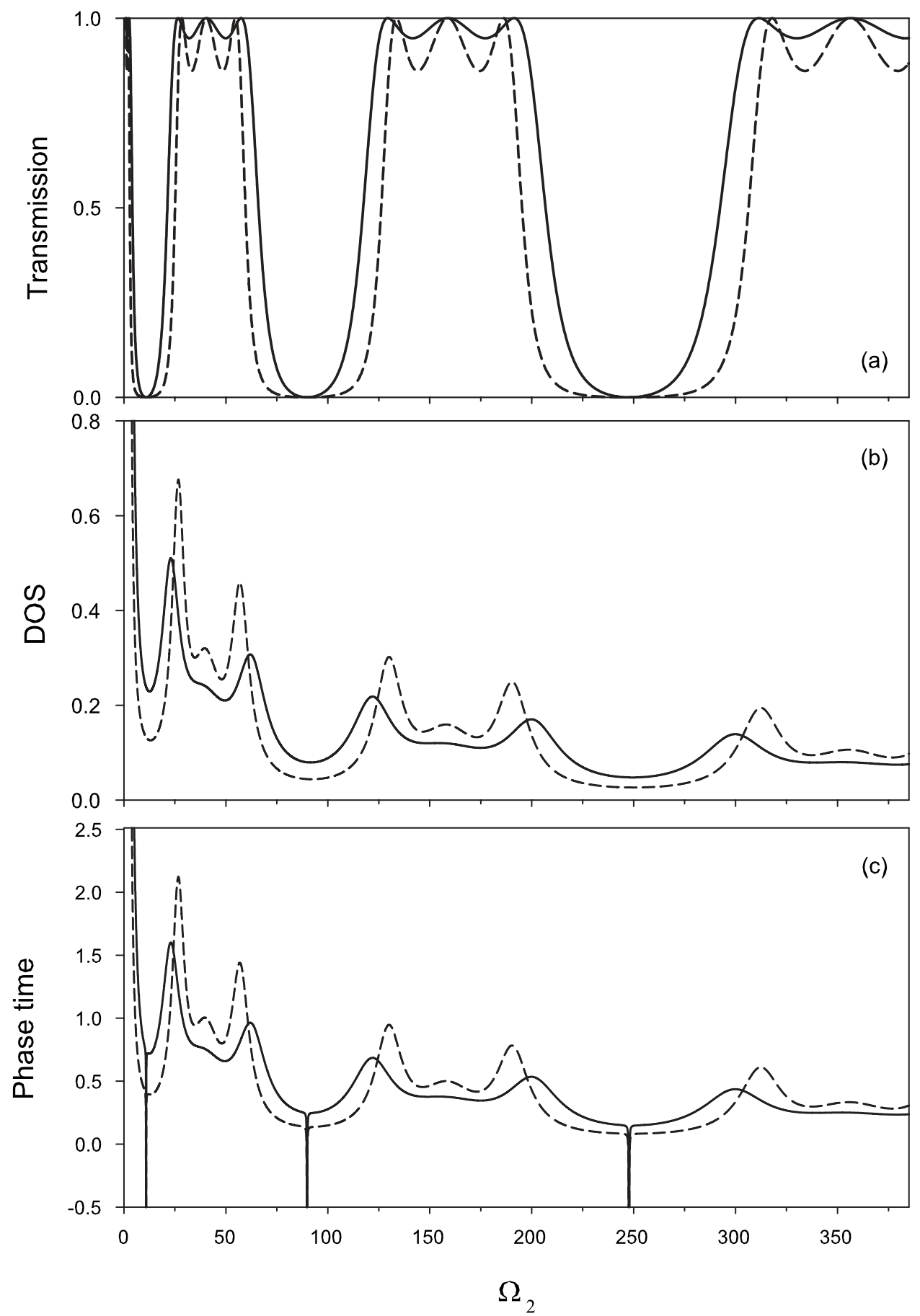

FIG. 6. (a) Transmission coefficient vs the reduced frequency $\Omega_{2}$ for the structures depicted in Fig. 1(c) (solid line) and Fig. 1(e) (dashed line). (b) The same as in (a) but for the variation of the density of states (in units of $\left.d_{2}^{2} / 2 D^{\prime}\right)$. (c) The same as in (b) but for the variation of the phase time (in units of $d_{2}^{2} / 2 D^{\prime}$ ). The parameters are $d_{1}=d_{3}=0.5 d_{2}$ and $\tilde{H}$ $=1$. zeros at $\Omega_{2}=10.87,89.3$, and 247.74 , according to Eq. (10). These delta functions have been enlarged by adding a small imaginary part to the pulsation $\omega$, which plays the role of absorption in the system. Such negative delta peaks have been shown experimentally in a simple photonic ${ }^{47}$ and phononic ${ }^{48}$ loop waveguide, giving rise to the so-called superluminal or negative group velocity. Figures 6(b) and 6(c) clearly show, in accordance with Eqs. (10) and (11), that except the frequencies lying around the transmission zeros, the DOS and the phase time exhibit exactly the same behavior.

\section{SUMMARY AND CONCLUSION}

In summary, we have clearly demonstrated that a simple geometry of a 1D mono-mode waveguide with dangling side resonators on both sides can pave the way to the derivation of gaps in the spin-wave propagation. The existence of the stop bands in the spectrum is attributed to the zeros of transmission associated with the dangling resonators. The width of the transmission gaps depends on the number of the side resonators grafted on both sides of the backbone. Besides the transmission gaps, we have shown the existence of asymmetric and symmetric Fano resonances that may lie near the 
vicinity of a transmission zero or be squeezed between two transmission zeros. These resonances are obtained by tailoring the lengths of the different branches constituting the structure and for different values $N=N^{\prime}$ of the DSBs. A study of the phase of the transmission function enables us to deduce several properties on the spin-wave propagation, through such structures, such as the phase times and therefore the density of states. The phase time calculation are, in general, the same as the density of states, except for the frequencies lying around the transmission zeros where the phase time may exhibit additional negative delta peaks.

The advantage of the simple magnonic waveguide model presented in this work consists in finding simple analytical expressions. These expressions enables us to discuss the existence of Fano resonances as well as the effect of the different segment lengths and the number of DSBs on tailoring these resonances without incorporating a defect in the structure as it is usually the case in the electronic counterparts studies. ${ }^{34-37}$ We believe that this paper brings a new piece of work in the field of spin-wave transport in 1D waveguide structures and we hope that it will stimulate an experimental observation of the transmission gaps and Fano resonances exhibited by the simple magnonic waveguide described in this work.

\section{ACKNOWLEDGMENTS}

H.A.-W. and E.H.E.B. gratefully acknowledge the hospitality of the Institut d'Electronique, de Microélectronique et de Nanotechnologie (IEMN), UMR CNRS 8520 and UFR de Physique, Université des Sciences et Technologies de Lille.
*Corresponding author; abdellatif.akjouj@univ-lille1.fr

${ }^{1}$ S. L. Vysotskii, S. A. Nikitov, and Yu. A. Filimonov, JETP 101, 547 (2005).

${ }^{2}$ S. A. Nikitov, Ph. Tailhades, and C. S. Tsai, J. Magn. Magn. Mater. 236, 320 (2001); Yu. V. Gulyaev and S. A. Nikitov, Dokl. Akad. Nauk 380, 469 (2001).

${ }^{3}$ J. O. Vasseur, L. Dobrzynski, B. Djafari-Rouhani, and H. Puszkarski, Phys. Rev. B 54, 1043 (1996).

${ }^{4}$ M. Krawczyk, J. C. Levy, D. Mercier, and H. Puszkarski, Phys. Lett. A 282, 186 (2001).

${ }^{5}$ A. Figotin and I. Vitebsky, Phys. Rev. E 63, 066609 (2001).

${ }^{6}$ D. S. Deng, X. F. Jin, and Ruibao Tao, Phys. Rev. B 66, 104435 (2002).

${ }^{7}$ L. Berger, Phys. Rev. B 54, 9353 (1996).

${ }^{8}$ J. C. Slonczewski, J. Magn. Magn. Mater. 159, L1 (1996).

${ }^{9}$ G. A. Prinz, Phys. Today 48(4), 58 (1995).

${ }^{10}$ See, e.g., The Handbook of Microlithography, Micromachining, and Microfabrication, edited by P. Rai-Chaudhry (SPIE, Bellingham, WA, 1996); X. F. Wang, P. Vasilopoulos, and F. M. Peeters, Phys. Rev. B 65, 165217 (2002).

${ }^{11}$ M. Krawczyk and H. Puszkarski, Cryst. Res. Technol. 41, 547 (2006).

${ }^{12}$ M. Krawczyk and H. Puszkarski, Acta Phys. Pol. A 93, 805 (1998).

${ }^{13}$ H. Puszkarski and M. Krawczyk, Solid State Phenom. 94, 125 (2003).

${ }^{14}$ Y. V. Gulyaev, S. A. Nikitov, L. V. Zhivotovskii, A. A. Klimov, P. Tailhades, L. Presmanes, C. Bonningue, C. S. Tsai, S. L. Vysotskii, and Y. A. Filimonov, JETP Lett. 77, 567 (2003).

${ }^{15}$ D. P. DiVincenzo and D. Loss, Superlattices Microstruct. 23, 419 (1998); B. E. Kane, Nature (London) 393, 133 (1998).

${ }^{16}$ H. Puszkarski and M. Krawczyk, Phys. Lett. A 282, 106 (2001).

${ }^{17}$ H. Puszkarski, M. Krawczyk, J.-C. S. Levy, and D. Mercier, Acta Phys. Pol. A 100, 195 (2001).

${ }^{18}$ V. V. Kruglyak and A. N. Kuchko, Phys. Met. Metallogr. 93, 511 (2002).

${ }^{19}$ V. V. Kruglyak and A. N. Kuchko, Physica B (Amsterdam) 339, 130 (2003).

${ }^{20}$ V. V. Kruglyak and A. N. Kuchko, J. Magn. Magn. Mater. 272-
276, 302 (2004).

${ }^{21}$ H. Al-Wahsh, A. Akjouj, B. Djafari-Rouhani, J. O. Vasseur, L. Dobrzynski, and P. A. Deymier, Phys. Rev. B 59, 8709 (1999).

${ }^{22}$ A. Akjouj, A. Mir, B. Djafari-Rouhani, J. O. Vasseur, L. Dobrzynski, H. Al-Wahsh, and P. A. Deymier, Surf. Sci. 482485, 1062 (2001).

${ }^{23}$ H. Al Wahsh, A. Akjouj, B. Djafari-Rouhani, A. Mir, and L. Dobrzynski, Eur. Phys. J. B 37, 499 (2004).

${ }^{24}$ A. Mir, H. Al Wahsh, A. Akjouj, B. Djafari-Rouhani, L. Dobrzynski, and J. O. Vasseur, Phys. Rev. B 64, 224403 (2001).

${ }^{25}$ A. Mir, H. Al-Wahsh, A. Akjouj, B. Djafari-Rouhani, L. Dobrzynski, and J. O. Vasseur, J. Phys.: Condens. Matter 14, 637 (2002).

${ }^{26}$ H. Al-Wahsh, A. Mir, A. Akjouj, B. Djafari-Rouhani, and L. Dobrzynski, Phys. Lett. A 291, 333 (2001).

${ }^{27}$ H. Al-Wahsh, Phys. Rev. B 69, 012405 (2004).

${ }^{28}$ A. Encinas-Oropesa, M. Demand, L. Piraux, I. Huynen, and U. Ebels, Phys. Rev. B 63, 104415 (2001); R. Arias and D. L. Mills, ibid. 63, 134439 (2001).

${ }^{29}$ For a recent discussion of this effect in the electronic counterparts, see H. Al-Wahsh, E. H. El Boudouti, B. Djafari-Rouhani, A. Akjouj, and L. Dobrzynski, Phys. Rev. B 75, 125313 (2007).

${ }^{30}$ C. Goffaux and J. Sánchez-Dehesa, Phys. Rev. B 67, 144301 (2003).

${ }^{31}$ C. Goffaux, J. Sánchez-Dehesa, A. L. Yeyati, P. Lambin, A. Khelif, J. O. Vasseur, and B. Djafari-Rouhani, Phys. Rev. Lett. 88, 225502 (2002).

${ }^{32}$ Z. Liu, C. T. Chan, and Ping Sheng, Phys. Rev. B 71, 014103 (2005).

${ }^{33}$ Y. A. Kosevich, C. Goffaux, and J. Sánchez-Dehesa, Phys. Rev. B 74, 012301 (2006).

${ }^{34}$ U. Fano, Phys. Rev. 124, 1866 (1961).

${ }^{35}$ D. Goldhaber-Gordon, H. Shtrikman, D. Mahalu, D. AbushMagder, U. Meirav, and M. A. Kastner, Nature (London) 391, 156 (1988); J. Gores, D. Goldhaber-Gordon, S. Heemeyer, M. A. Kastner, H. Shtrikman, D. Mahalu, and U. Meirav, Phys. Rev. B 62, 2188 (2000); Y. Ji, M. Heiblum, and H. Shtrikman, Phys. Rev. Lett. 88, 076601 (2002).

${ }^{36}$ K. Kobayashi, H. Aikawa, S. Katsumoto, and Y. Iye, Phys. Rev. 
Lett. 88, 256806 (2002); J. Kim, J.-R. Kim, Jeong-O Lee, J. W. Park, H. M. So, N. Kim, K. Kang, K.-H. Yoo, and J.-J. Kim, ibid. 90, 166403 (2003); B. Babic and C. Schönenberger, Phys. Rev. B 70, 195408 (2004).

${ }^{37}$ Y. S. Joe, A. M. Satanin, and G. Klimeck, Phys. Rev. B 72, 115310 (2005).

${ }^{38}$ M. L. Ladrón de Guevara, F. Claro, and P. A. Orellana, Phys. Rev. B 67, 195335 (2003); K. K. Voo and C. S. Chu, ibid. 72, 165307 (2005); G. Hackenbroich and H. A. Weidenmuller, Phys. Rev. Lett. 76, 110 (1996).

${ }^{39}$ J. Wu, B. L. Gu, H. Chen, W. Duan, and Y. Kawazoe, Phys. Rev. Lett. 80, 1952 (1998); T. Taniguchi and M. Buttiker, Phys. Rev. B 60, 13814 (1999); H. W. Lee, Phys. Rev. Lett. 82, 2358 (1999).

${ }^{40}$ A. Yacoby, M. Heiblum, D. Mahalu, and H. Shtrikman, Phys. Rev. Lett. 74, 4047 (1995); R. Schuster, E. Buks, M. Heiblum, D. Mahalu, V. Umansky, and H. Shtrikman, Nature (London) 385, 417 (1997).

${ }^{41}$ J. O. Vasseur, A. Akjouj, L. Dobrzynski, B. Djafari-Rouhani, and
E. H. El Boudouti, Surf. Sci. Rep. 54, 1 (2004).

${ }^{42}$ M. G. Cottam and A. A. Maradudin, Surface Excitations, Modern Problems in Condensed Matter Sciences Vol. 9 (NorthHolland, Amsterdam, 1984).

${ }^{43}$ L. Dobrzynski, Surf. Sci. Rep. 11, 139 (1990).

${ }^{44}$ M. Büttiker and R. Landauer, Phys. Rev. Lett. 49, 1739 (1982); M. Büttiker, Phys. Rev. B 27, 6178 (1983); E. H. Hauge and J. A. Stovneng, Rev. Mod. Phys. 61, 917 (1989).

${ }^{45}$ M. L. H. Lahlaouti, A. Akjouj, B. Djafari-Rouhani, L. Dobrzynski, M. Hammouchi, E. H. El Boudouti, A. Nougaoui, and B. Kharbouch, Phys. Rev. B 63, 035312 (2001).

${ }^{46}$ See, for example, W. Porod, Z. Shao, and C. S. Lent, Appl. Phys. Lett. 61, 1350 (1992); Phys. Rev. B 48, 8495 (1993).

${ }^{47}$ E. H. El Boudouti, N. Fettouhi, A. Akjouj, B. Djafari-Rouhani, A. Mir, J. Vasseur, L. Dobrzynski, and J. Zammouri, J. Appl. Phys. 95, 1102 (2004).

${ }^{48}$ W. M. Robertson, J. Pappafotis, and P. Flannigan, Appl. Phys. Lett. 90, 014102 (2007). 